Gefässchirurgie 2016 $21: 536-537$

DOI 10.1007/s00772-016-0220-0

๑) Springer Medizin Verlag Berlin 2016

CrossMark

\title{
A. Larena-Avellaneda
}

Klinik und Poliklinik für Gefäßmedizin, Universitäres Herzzentrum Hamburg, Gefäßchirurgie Endovaskuläre Therapie - Angiologie, Universitätsklinikum Hamburg-Eppendorf, Hamburg, Deutschland

\section{Herausgebersitzung - Postskriptum}

Die Kosten hierfür trägt dann der $\mathrm{Au}-$ tor, die Gebühren liegen bei $2200 €$ zzgl. MwSt. Hierbei ist aber eines zu berücksichtigen: Die „Open-choice“-Publikation eines Artikels aus der Gefässchirurgie soll helfen, den Artikel auch international auffindbar und damit zitierbarer zu machen. Der Journal-Impact-Faktor beruht auf diesen Zitationen. Aus zwei Gründen ist es sehr sinnvoll, einen Artikel nur in Englisch als „Open-choice“-Artikel zu publizieren: Erstens werden internationale Autoren den deutschen Artikel nicht lesen und damit auch nicht zitieren. Zweitens, und dass ist noch wichtiger, werden nur die englischsprachigen „Openchoice"-Artikel automatisch in PubMed Central gesetzt und somit in PubMed auffindbar. Anders formuliert: Ein deutschsprachiger „Open-choice“-Artikel wird nicht in PubMed zu finden sein.

Es besteht allgemeiner Konsens im Herausgebergremium, dass das „Openaccess“- bzw. „Open-choice“-Konzept als Hybrid-Zeitschrift der Weg sein soll, um international gesehen und zitiert zu werden. Daher haben sich die 3 Fachgesellschaften DGG, ÖGG und SGG sowie der Springer Medizin Verlag bereit erklärt, für 8 Artikel eine solche Publikation zu finanzieren. Der bisher vereinbarte Zeitraum von 18 Monaten erscheint aber nicht ideal, sodass angestrebt wird, 8 Artikel pro Jahr, also einen Artikel pro Ausgabe „open choice“ zu setzen. Die Auswahl des Artikels obliegt der Schriftleitung und den (Heft-)Herausgebern. Der Artikel wird dabei ins Englische übersetzt, d. h. in der gedruckten Zeitschrift erscheint er in deutscher Originalversion, während die englische Übersetzung als „Open-choice“-Artikel online publiziert wird.
Neben diesen 8 finanzierten Artikeln besteht aber für jeden Autor die Möglichkeit, seine Ausarbeitung sekundär in Englisch selbst „open choice“ zu setzen. Die Kosten habe ich oben genannt. Sollten Sie diese Möglichkeit nutzen wollen, so wäre es sehr sinnvoll, dies mit der Schriftleitung abzusprechen, damit nicht die deutsche Version, sondern die englische Übersetzung sichtbar wird.

Ziel ist wie gesagt, vermehrt international zitiert zu werden. Neben den „Open-choice“-Artikeln können Sie, verehrte Autorinnen und Autoren, dieses Anliegen ebenfalls unterstützen, indem Sie geeignete Artikel aus der Gefässchirurgie (wichtig: der letzten beiden Jahre, also 2015 und 2016) in Ihren international publizierten Beiträgen berücksichtigen.

Ob dieser Weg am Ende funktioniert, und wir den Impact Faktor wieder beantragen können, wissen wir noch nicht. Es fällt aber auf, dass alle 3 in 2015 in englischer Sprache publizierten „Openchoice“-Artikel bereits in hochrangigen, internationalen Zeitschriften zitiert wurden.

Ich hoffe sehr, dass ich Ihnen die Abläufe verständlich näher bringen konnte und freue mich weiterhin auf qualitativ hochwertige Einsendungen.

Mit herzlichen Grüßen, Ihr

Axel Wuena - Nellende

Prof. Dr. A. Larena-Avellaneda 


\section{Korrespondenzadresse}

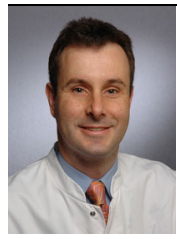

Prof. Dr. A. Larena-

Avellaneda

Klinik und Poliklinik für

Gefäßmedizin, Universitäres

Herzzentrum Hamburg,

Gefäßchirurgie - Endovaskuläre Therapie - Angiologie,

Universitätsklinikum

Hamburg-Eppendorf

Martinistraße 52, Gebäude

Ost 70, 20246 Hamburg,

Deutschland

larena@uke.de

Interessenkonflikt. A. Larena-Avellaneda gibt an, dass kein Interessenkonflikt besteht.

\section{Julius-Springer-Preis für Gefäßmedizin 2017}

Der Brückenschlag von Forschungsarbeit zum klinischen Nutzen wird ausgezeichnet

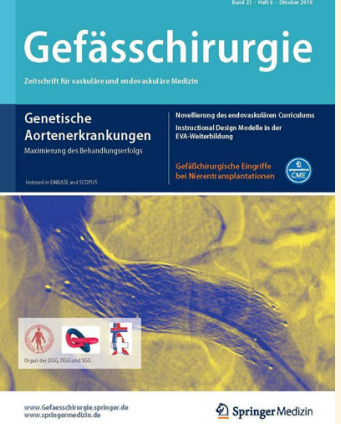

Die Gefäßmedizin ist ein Wachstumsfach mit zunehmender Bedeutung im Gesamtkonzept der Diagnostik und Therapie von Gefäßerkrankungen. Die wissenschaftliche Leitung von Gefässchirurgie aus Mitgliedern der deutschen, österreichischen und schweizerischen Gesellschaften für Gefäßchirurgie möchte zusammen mit dem Springer Medizin Verlag die wissenschaftliche Forschung auf diesem Gebiet unterstützen und insbesondere den Nachwuchs durch die Verleihung des 8. Julius-Springer-Preises für Gefäßmedizin fördern.

Nutzen Sie die Gelegenheit und reichen Sie ein Exposé bis zum 15. August 2017 ein.

Der Preisträger erhält einen Geldpreis in Höhe von $500 €$

\section{Teilnahmebedingungen}

- Der Autor soll ein Exposé auf Grundlage einer eigenen klinischen oder experimentellen Arbeit verfassen. Die Jury legt besonderen Wert auf den edukativen Charakter des Artikels sowie die Herausarbeitung der klinischen Relevanz - der Beitrag soll eine Brücke von den erhobenen Daten zur klinischen Anwendung schlagen.
- Der Sieger wird gebeten, einen Übersichtsbeitrag in deutscher Sprache zu verfassen, der in Gefässchirurgie publiziert wird.

- Alle anderen Autoren sind frei, ihren Beitrag anderweitig zu publizieren.

Der Siegerbeitrag wird durch eine Jury aus dem Herausgebergremium unter Regie der Schriftleitung bestimmt

Preisverleihung im Rahmen der 33. Jahrestagung der DGG

Die Verleihung des 8. Julius-Springer-Preises für Gefäßmedizin erfolgt im Rahmen der 33 Jahrestagung der Deutschen Gesellschaft für Gefäßchirurgie und Gefäßmedizin vom 27.-30. September 2017 in Frankfurt/Main.

Bitte reichen Sie Ihr Exposé in elektronischer Form bei der Verlagsredaktion bis zum 15. August 2017 ein:

sara.weber@springer.com 